\section{Pediatric diaphragmatic pacing}

\author{
Tarek R. Hazwani, Bedoor Alotaibi, \\ Wadha Alqahtani, Asma Awadalla, \\ Ali Al Shehri
}

\section{King Abdullah Medical Research Center, King Saud bin Abdulaziz University for Health Sciences, King Abdulaziz Medical City, Ministry of National Guard-Health Affairs (NGHA), Riyadh, Saudi Arabia}

\begin{abstract}
Diaphragmatic pacing has been shown to play a significant role in adult patients with diaphragmatic paralysis and facilitates mechanical ventilation weaning. However, reports on its use in paediatric patients are scarce. This report is about a 4-year-old child with a spinal cord injury secondary to a motor vehicle accident that led to quadriplegia and diaphragm paralysis. The patient underwent a diaphragmatic pacing procedure, which helped start gradual weaning from mechanical ventilation. We reviewed the concept of the diaphragmatic pacer and its types depending on the site of the implantation. In addition, we reviewed who can benefit from using a diaphragmatic pacer and compared its use in adults versus paediatric patients. Our case showed that diaphragmatic pacing appears to be effective, as it facilitates mechanical ventilation weaning and improves the quality of life outcome in paediatric patients with diaphragmatic paralysis.
\end{abstract}

\section{Introduction}

Although a mechanical ventilator has beneficial outcomes over a long period of time, it can lead to recurrent respiratory infection, loss of the sense of smell, difficulties with moving or transferring patients, loss of the ability to talk, and a decrease in mobility that further negatively affects body functioning. ${ }^{1}$ Here, we present the concept of diaphragmatic pacing (DP) to overcome the use of a mechanical ventilator and to reduce its harmful effects in patients with diaphragmatic paralysis. ${ }^{1}$

DP uses electrical impulses to the phrenic nerve in order to produce artificial respiration, and it was a well-known concept in the $18^{\text {th }}$ century although without clinical applications. This concept was then technically applied in the $19^{\text {th }}$ century. ${ }^{2-7}$ A diaphragmatic pacer is defined as a device that uses electrical signals to stimulate the diaphragm to contract in order to restore normal breathing mechanisms and to reduce mechanical ventilation dependence in patients with diaphragmatic paralysis. ${ }^{8}$

A diaphragmatic pacer works by increasing tidal volume as much as possible by changing different characteristics of the breath cycle in a patient with diaphragmatic paralysis.

DP is classified into two groups depending on the implantation site. The device is either implanted exactly on the phrenic nerve or placed directly on the diaphragm muscle. ${ }^{8}$ Patients with respiratory insufficiency, spinal cord injury, stroke or any defect in the medulla mostly have beneficial outcomes from the diaphragmatic pacer. 9 Other reported indications include chronic obstructive pulmonary disease (COPD), spinal muscular atrophy, idiopathic diaphragmatic paralysis, non-progressive neuromuscular diseases and chest deformities. ${ }^{2}$ The diaphragmatic pacer can be implanted for patients with spinal cord injury as well, but the level of injury can determine whether the patient is ready to have diaphragmatic pacer placement or not. Thus, patients with injury at the level of $\mathrm{C} 3$, $\mathrm{C} 4$ and $\mathrm{C} 5$ cannot be paced because of the effects on the phrenic nerve.

Diaphragmatic pacing has been shown to play a significant role in adult patients. Christopher et al. studied implantation of a diaphragm pacer in patients with different diseases and showed that they had a high survival rate and better quality of life. $10 \mathrm{On}$ the other hand, reports on paediatric patients with diaphragmatic paralysis who underwent implantation of a diaphragmatic pacer are not common. In addition, there are very few reports that compare paediatric cases with adult cases.

\section{Case Report}

Our case involves a 4-year-old child who was referred to our hospital in May 2016 for spinal surgery secondary to a motor vehicle accident 18 days before that resulted in upper cervical cord contusion with an injured apical ligament. This led to quadriplegia and diaphragm paralysis, for which the patient underwent posterior occipitocervical fixation.

After fixation surgery, the patient stayed in the Paediatric Intensive Care Unit (PICU) for a long time since he became ventilator-dependent secondary to his diaphragm paralysis. Then, he was moved to the high dependency unit with his home ventilator. He was completely quadriplegic without any signs of significant improve-
Correspondence: Tarek Hazwani, King Abdullah Medical Research Center, King Saud bin Abdulaziz Medical City, Ministry of National Guard-Health Affairs (NGHA), P.O.Box 22490 , Riyadh 11426, Kingdom of Saudi Arabia.

Tel.: +966.118011111/52457

Fax: $+966.118011111 / 53555$.

E-mail: tarekhiz@yahoo.com

Key words: Children; diaphragm; pacing; spinal cord injury; phrenic paralysis; diaphragmatic paralysis; spinal cord stimulator.

Contributions: the authors contributed equally.

Conflict of interests: the authors declare no potential conflict of interests.

Funding: None.

Received for publication: 17 December 2018. Accepted for publication: 22. February 2019.

This work is licensed under a Creative Commons Attribution NonCommercial 4.0 License (CC BY-NC 4.0).

(C) Copyright T.R. Hazwani et al., 2019

Licensee PAGEPress, Italy

Pediatric Reports 2019; 11:7973

doi:10.4081/pr.2019.7973

ment; however, he was mentally intact and communicated by eye movement. Thus, a multidisciplinary medical decision had been made for diaphragmatic pacing, which was done 14 months following the primary injury. Weaning from the mechanical ventilator was not smooth due to a discomforting sensation from pacing pulses during the conditioning phase, and so the pacing device was set at low amplitude initially, 10 $\mathrm{mA}$ (milliampere), and pulse width of 110 $\mu \mathrm{S}$ (microseconds), after which it was increased gradually until it reached an amplitude of $20 \mathrm{~mA}$ and pulse width of 150 $\mu \mathrm{S}$. These steps were associated with weaning from a mechanical ventilator by increasing the free ventilator time gradually until it reached 8 hours in the daytime.

Currently, he is on a pacer for only eight hours in the daytime and on a home ventilator without the pacer for the remaining 16 hours. The medical team's plan was to increase pacing time gradually.

\section{Discussion}

To our knowledge, the majority of the studies on DP in the literature review are related to adults and not paediatric patients. Regarding studies that have been reviewed, 
most adult patients tolerate diaphragmatic pacing and can be easily weaned off the mechanical ventilator in a short time. In general, outcomes of DP in adults are better than those in paediatric patients. Oakes DD performed a study on 11 adult patients with implantation, of which 3 became completely independent of their ventilators in three months. ${ }^{11}$ Weaning from the mechanical ventilator in paediatric patients was not smooth, and most of the patients reached the maximum 10 hours off the ventilator, which occurred one year after the procedure. ${ }^{8}$

Looking at our case, we started with continuous pacing initially with gradual ventilator weaning by increasing the ventilator off-time. Due to intolerance of the electrical sensation produced by the pacer and sleeping disturbance, the pacer was only used during the daytime and the weaning strategy was started with 5 minutes only. Then, this time was increased gradually while only the ventilator was used for the remaining time. Week by week, this time was increased until a time of 8 hours off the ventilator with pacing was reached, and the patient shifted again to the mechanical ventilator for the rest of the day. This weaning achievement took five months after the procedure. With gradual weaning from the mechanical ventilator, we look forward to achieving our goal to liberate patients completely from ventilator dependency.

\section{Conclusions}

Our report demonstrates the safety of diaphragmatic pacing (DP) in children, which appears to be effective in patients with diaphragm paralysis. In addition, we found that DP facilitates mechanical ventilation weaning and improves quality of life in paediatric patients. Nonetheless, further studies are needed to validate the long-term safety and outcome of DP.

\section{References}

1. Onders RP, Ponsky TA, Elmo M, et al. First reported experience with intramuscular diaphragm pacing in replacing positive pressure mechanical ventilators in children. J Pediatr Surg 2011;46:726.

2. Chervin RD, Guilleminault C. Diaphragm pacing for respiratory insufficiency. J Clin Neurophysiol 1997;14:369-77.

3. Glenn WW, Brouillette RT, Dentz B, et al. Fundamental considerations in pacing of the diaphragm for chronic ventilatory insufficiency: a multi-center study. PACE 1988;11:2121-7.

4. Glenn WW, Hogan JF, Loke JS, et al. Ventilatory support by pacing of the conditioned diaphragm in quadriplegia. New Engl J Med 1984;310:1150-5.

5. Glenn WW, Hogan JF, Phelps ML.
Ventilatory support of the quadriplegic patient with respiratory paralysis by diaphragm pacing. Surg Clin North Am 1980;60:1055-78.

6. Glenn WW, Phelps ML. Diaphragm pacing by electrical stimulation of the phrenic nerve. Neurosurgery 1985; 17:974-84.

7. Glenn WW, Phelps ML, Elefteriades JA, et al. Twenty years of experience in phrenic nerve stimulation to pace the diaphragm. PACE 1986;9:780-4.

8. Filho Pinto DR, Tedde ML, Avino AJ, et al. Video-assisted thoracoscopic implantation of a diaphragmatic pacemaker in a child with tetraplegia: indications, technique, and results. J Bras Pneumol 2015;41:90-4.

9. Son BC, Kim DR, Kim IS, Hong JT. Phrenic nerve stimulation for diaphragm pacing in a quadriplegic patient. J Korean Neurosurg Soc 2013;54:359-62.

10. McDermott CJ, Maguire C, Cooper CL, et al. Protocol for diaphragm pacing in patients with respiratory muscle weakness due to motor neurone disease (DiPALS): a randomised controlled trial. BMC Neurol 2012;12:74.

11. Oakes DD, Wilmot CB, Halverson D, Hamilton RD. Neurogenic respiratory failure: a 5-year experience using implantable phrenic nerve stimulators. Ann Thorac Surg 1980;30:118-21. 